\title{
Heteronomy and (un)sustainability of essential technical infrastructures
}

\author{
A. van Timmeren ${ }^{1,2}$ \\ ${ }^{1}$ Climate Design \& Environment (CD\&E), \\ Delft University of Technology (TUD), Faculty of Architecture, \\ The Netherlands \\ ${ }^{2}$ Atelier 2T, Haarlem, The Netherlands
}

\begin{abstract}
The methods and techniques applied in present-day essential infrastructures for energy and sanitation supply may be considered traditional and driven by the separation and centralization paradigm. There is physical expansion and this is a structural phenomenon because of globalization combined with the liberalization of the energy market and - to a lesser extent - the solid-waste market. Moreover, there is growing heteronomy of the essential utilities, particularly energy and sanitation. There is a considerable increase in the electrification of society. For solutions to new or existing problems, technological development is following the "roads present": the existing paradigm. Strict rules and regulations often make this necessary. Changes, innovations or adaptations within these essential (infra)structures follow the principles of the quasi-evolutionary model, while during the last few years developments have more and more arisen from the endogeneous point of view and a certain kind of "techno-fix" cannot be denied. Little or nothing is done about the underlying causes of the environmental problems, whereas unforeseen side effects occur, e.g. a large amount of strongly polluted sludge in waste water treatment.

Many relevant participants do not seem to realize that other, more sustainable alternatives can be found by abandoning the specific characteristics of the traditional paradigms rather than following them. This paper emphasises the potentials for sustainability and resilience in case of a reciprocal relation between centralized and decentralized systems and the interconnection of energy, waste and wastewater solutions.
\end{abstract}

Keywords: heteronomy, infrastructures, autonomy, integration strategy. 


\section{Introduction}

Where the essential infrastructures are concerned, the liberalization of the markets shows that the goals set cannot always be accomplished in an integral way. At a national level, there is (still) too little grip on the developments. The demand for supervision or rules at a supra-national (European) level is being heard, and this causes one of the two main reasons for this liberalization to be surpassed. Changes in technological choices and in the framework are based on political decisions and (market-driven) sectoral strategies. This asks for target values and conditions to be formulated. Market participants have no interest in overcapacity, which puts pressure on the reliability of supply (by a maximum bid on the available capacity).

Pressure can also be put on the other long-term interests, including maintenance of grids and investments in, research into or application of innovations (other techniques, other subsystems or even other (infra)structures and technology). Other aspects (for the users) are sustainability, a guarantee on supply and processing and affordability.

For sectors that are left to market forces, positive effects are soon to be expected on the efficient use of the (infra)structures by oligopolistic market types, and, thus, on the affordability of the accompanying services. There will be a (well-known) dilemma between the short term (economic efficiency) and the long term (sustainability and guarantee of supply).

In the Netherlands, the contributions to energy independence and knowledge infrastructure of the country are tasks for the government, or, in other words, a "the public interest". Nevertheless, it seems reasonable that the dominant market participants also subscribe to these strategic targets, or at least facilitate them. Politics still aims too much for certainties and guarantees in advance. Given the nature of the changes (particularly in the internationalizing markets), politics should pay more attention to the uncertainties and, consequently, to the question of how public interests can be prevented from getting stuck in case of unforeseen and undesired developments.

Convergence is a new characteristic of the various technical infrastructures. It results in greater complexity and more dependence on the structures as perceived by users. Reliability and - in an indirect way - affordability gain more importance. At this moment, this is still at the expense of sustainability. This may be overcome by including sustainability, via reliability, as an added value at relatively little cost, e.g. in the form of a decentralized backup. Too little advantage is taken of this subaspect of sustainability. For small-scale users, this results in a simpler arena, particularly where the number of parties is concerned with which contracts have to be signed. Other characteristics of development according to the traditional centralization paradigm of the essential technical infrastructures are: specialization and segmentarization, with one or several dominant parties per subflow or sector as major results. The dominant participants have an interest in using existing structures as efficiently as possible and in developing them further with as few risky investments as possible. As yet, 
the cost of transport (distances) is not taken into account, and there is little product differentiation.

As a result of the increasing importance of flexibility, sustainability and certainties, it is evident that the call for solutions according to the present "integral" approach (with the aid of a source-focused and preventive policy as an important condition for all built-up areas) adds too little value as either a process guiding or process following interpretation. With administrative-organizational "integralness" as a starting point, there is too much focus on the advantages of business economics resulting form horizontal or vertical integration. "Integralness" is used too often and, as a result, is becoming a container notion, possibly even an empty paradigm.

The current paradigm, specified by the participants connected with the essential flows and infrastructures, seems to aim at a development following the principle of the "economies of scale". In addition to more far-reaching interconnection, this implies a vertical integration building on administrativeorganizational integralness. In this process of interconnection and integration, the aspects discussed, viz. certainty and sustainability, will eventually be normative for a well-considered choice of interpretation. Here, the so-called physical integralness will be of increasing importance.

The presented research tries to demonstrate the need to include interdisciplinary approaches to the integration of strategies for raising public awareness, marketing of the different qualities of water (cascading) and energy (exergy), and establishing a service business for building and operating more decentralized installations. The general aim is the integration strategy for water management and sanitation (healthy wastewater and waste streams) together with energy-generation at scales nearer to users or residents. On the one hand it comprises direct linking of building and urban settlement with their surroundings and/or (green) hinterland. On the other hand with neighboring subjects like agriculture (especially urban farming), aquaculture, horticulture, health care and food security.

The research has been commissioned by the Delft University of Technology (TUD) as part of the DOSIS (Sustainable Development of City \& InfraStructures) project recently continued in CD\&E - Climate Design \& Environment - research to investigate and develop decentralized sanitation, energy and reuse technologies. The aim is to research the spatial, social and environment related consequences of the implementation of decentralised technologies, and to define the conditions within society, with emphasis on urban planning and building.

\section{Heteronomy and the effects on sustainability}

\subsection{Sustainability of the essential technical infrastructures}

It is important to distinguish between underground and aboveground infrastructures. As yet, there is little knowledge of environmental costs of the technical, often underground, infrastructure. It is not known how the 
relationships between the infrastructure inside the building, and the infrastructure systems outside the building and in the area in between can affect the environmental effects (or environmental costs) as a collective process.

Since much is known about the sustainability of the infrastructure and facilities at the level of the building, it has been decided to test the environmental costs at the next levels, i.e. the ones higher than this smallest relationship. In this case, this is the level of the (city) district and/or neighbourhood. For the visualization of the environmental load of a prototypal reference project, this research studies part of the development Oosterhout in Nijmegen (the Netherlands). The modelling and calculation program Greencalc was used, that translates the environmental load into environmental costs. In this new housing estate (finished in 2001), the environmental load of the technical infrastructure (excluding roads) is $10.4 \%$ of the total environmental load. This portion may be considered non-significant, but at the same time it is not to be neglected, particularly where the hot-water infrastructure and sewage infrastructure are concerned.

In three alternative configurations of the reference case of Oosterhout, it turned out that a reduction of the technical infrastructure of heat supply (which was the one with the highest environmental load) at the level of the district did not automatically lead to a lower total environmental load of the district. An example is the configuration based on a natural gas grid with individual heat supply (boilers) instead of the shared heating grid. The alternative configuration using heat pumps did produce a (slight) reduction of the total environmental load.

In general, the calculation of environmental costs of the technical infrastructure of this new district in Nijmegen and the related techniques prove that the smallest amount of environmental load occurs in high-efficiency heating systems with generation close to the user. More important, however, is that reductions based on optimized urban development structures have a larger effect on the environmental load than optimizing the infrastructure using other system or transport options.

Hence, optimization of central utilities or other facilities demands "compact building".

\section{2 (Further) preservation}

Looked on from the social aim of "sustainable development", the path of expansion selected is not necessarily the optimum as perceived subjectively. A characteristic of expansion is the increasing importance of relocating the material and energy flows. In this, physical infrastructures play an important part. They are the bases for the supply of processes, products and services that meet the fundamental needs. Building infrastructure almost always implies slow and large-scale processes in the "underground" layer. For a structural solution and preservation, the technical infrastructure should be considered, as the lowest layer in this model of layers. It will be leading for the design and the allocation of the faster dynamics of the overlying layer: the layer of the "networks" and that of "occupation". 
The infrastructure strongly correlates with production (supply as well as drainage). A change desired in the infrastructure, e.g. a bottleneck with respect to capacity, can be solved by investing in extending the infrastructure (now often accepted), but often also by adapting the "production" in strategic spots of the (central) grid. One possibility is connecting or disconnecting (decentralized, additional) sustainable subproduction (generation or processing capacity). This may imply a gradual change of the paradigm, following a sliding time scale rather than a radical change at a certain, perhaps unexpected, point in time. Moreover, it may involve short-term interventions for long-term guarantees (sustainability, guarantees for supply or processing and affordability). Such a principle may be useful as a kind of fall-back scenario for, for example, a serious and unforeseen dysfunction of the current process of further scaling up and liberalization of sectors.

\subsection{Introduction of decentralized systems}

There are clear differences between the characteristics (or rather: advantages and disadvantages) of the various central networks, in the energy and sanitation subflows each as well as between the energy and sanitation supply as a whole. They are caused by different "central scales" of application and different extents of visibility, but also by the management structure and the presence or absence of liberalization processes. The increasing heteronomy observed in the so-called "essential" networks and accompanying managing parties for end users does not only hold for central networks, but also for decentralized or local systems. The difference lies in the consequences of catastrophes and the extent of visibility (the subjective perception) of the results of this heteronomy for the end user.

There is a common consensus in society about the necessity of fundamental facilities for meeting the most fundamental needs in the own living environment, viz. "Maintenance", the so-called primary necessity of life. The availability of energy and food, including clean drinking water, and the removal of waste are parts of it. It is no use trying to introduce sustainability measures that harm this fundamental need. It has turned out that the ongoing processes of liberalization have put pressure on the importance of the certainty of supply, and sometimes also removal. Working certainty of supply and independence out in further detail seems necessary, or even essential, not only for further development based on the future of scaling-up ("economies of scale"), but also for decentralization ("scale economy").

The distance created between the (environmental) problem and its solution leads to more and more complexity. The process of changing the interrelated public and private services, systems and infrastructures is becoming more and more complicated and less and less predictable. Together with the increased scaling, the interconnection of the various flows and the growing number of parties and techniques involved has increased the end users' (consumers') (subjective) dependence. This asks for a simplification of the processes, products (or rather: services) and parties involved. A larger concentration on integral provision of services, or, in other words, the supply and management of integral 
packages, offers possibilities. Also the level of application should attune better to the lifestyle and direct surroundings of the users.

The ongoing individualization more and more often leads to a pursuit of decreased independence on public infrastructures and the wish for decentralized utilities (connected or not), with autonomy of the individual or the household as an extreme version. A decentralized system must not be characterized as a static system, since there is an ongoing change of an existing situation. The scale level of a decentralized system is relatively fixed. It depends on the technique of the administrative body itself, the context and the position of the observer. Technical (de)centralization concerns (a change of/in) systems. In the case of administrative decentralization, there is a distinction according to the nature of the administrative bodies: territorial decentralization (between/carried out by Government, Province and Municipality) and functional decentralization (within the Municipality).

As for technical decentralization, the various flows have different definitions of (the scale of) subclusters and of "decentralized" subnetworks and subsystems. Often, there is vagueness even within the various flows. The scale level is considered decentralized, but is defined in a relative manner too often. Regarding technical decentralization, this paper starts from the production and processing of the various flows closer to the users than is usually done, with the flows being fed back to the users in a direct way.

In administrative decentralization, the "sandwich strategy" may be a good starting point for the far-reaching support of making the various (infra)structures within town and country planning sustainable and possibly independent. Decentralized initiatives to solutions and environment-friendly behaviour are emphasized here.

Because of the relative new market of (technical) decentralization, "niches" can be created. It has turned out that this has happened before in history. Often, niches cause a set of instruments to be developed for the start of a new paradigm or system of techniques. With the aid of strategic niche management, innovations are implemented in this type of "sheltered area", tested and evaluated. It is possible that the creation of niches can also take place in a planned way. This is called "strategic niche management". The difference with the more familiar principle of "pilot projects" is that a shelter is built around the new technology in the case of strategic niche management, through which the technology can develop from prototype to an actually applicable technology. Eventually, the technology should work without any protective measures at all.

Generally speaking, the two main problems in decentralized solutions are scepticism of the leading (often dominant) parties involved and the larger influence of a fluctuating flow size. The former is particularly caused by responsibility (certainty) and liability. This scepticism will increase because of the necessary transition of the market(s) from supply of products to supply of services. The aspect of the flow size (in fact, the basis for the technical "economies of scale") can be met locally by modern techniques of control and tuning, the so-called "Real Time Control", and the subdivision into parallel facilities. Thus, the remaining main points of interest for improving the 
competitiveness of decentralized systems and actually achieving the advantages for the environment and the users are the organization and implementation of maintenance, exploitation, provision of services and inspection of the various systems, together with the availability of backup provisions if necessary.

\section{Alternative network geometry}

\subsection{Introduction}

For the essential (technical) infrastructure, the dynamics of non-simultaneous, slow transformation necessary for attuning the complex structures of society, the "flows" and nature (or natural processes) implies that it is wrong to still think in separate systems within integral development processes. That is, since there is an increasing interconnection and interdependence in the technical infrastructure of the essential flows. This does not concern local interconnections only. In fact, the total human system all over the world is linked with the issue of to what extent the increasing rate and complexity of change is integrated in a determined effort.

Two development processes concerning decentralized technology for the purpose of autonomy have come forward as topical: viz. first, the efficiency and improvements in the integration of subtechniques and co-ordinated, connected concepts, and, second, a better harmony between supply (input) and demand of the (different) subflows.

Additionally, there are two more general underlying development processes. The first is the environment-technical, environmental and, to some degree, also social optimization of decentralized systems within semi-autarkic projects. In spite of the potential of the underlying optimization principle of the "scale economy" claimed in much of the literature, and in spite of its importance, which was also proven, it has only been applied to a small extent. Consequently, there are not many "economies of scale". However, the subaspects concerning the application freedom and environmental integration (smaller sizes, fewer secondary demands, etc.) and user-related demands (comfort, ease of use, costs, etc.) do improve noticeably.

The second underlying development process concerns the link to economic applications related to the surroundings, often determined by soil or users, including taking nutrients back to agriculture and other lateral applications or possibilities, such as car-sharing systems. In addition to the possibility of other types of use of (agricultural) grounds (urban agriculture), the link to agriculture may not only lead to a structurally different infrastructure (aboveground and underground), but also to different country planning as a whole, when applied on a larger scale. This offers points of departure for interrelating "red" and "green" functions in environmental planning. Here, the aspects of vicinity and comfort are leading. In this situation, the search for an optimum scale of autarky or autonomy of the various essential subflows in the built-up environment gains higher importance. 
The changed network philosophy described as desired has far-reaching consequences for the way in which these infrastructures are designed and integrated.

For complex systems, the coherence with which and the way in which dynamic processes are dealt with determines the translation to physical "integralness".

It is important to establish that the stability or resilience of networks is directly related to their complexity. It is not the components of the various structures that matter, but the way they are organized together as intelligent structures. It is important to learn from the organization structure and topology of existing adaptive, complex structures. Recognizing the structures of each network is needed for combining their optimally ongoing development, possible decline and damage done to them, whether desired or not, with constant or increasing sustainability and certainty guarantees for users.

\subsection{Decentralization for the purpose of scale invariance}

In current central infrastructures of energy as well as waste water flows, the possibilities of an alternative network layout are not or not sufficiently taken into account. More and more connections are made between the various (national) networks and subnetworks in gas and electricity networks, but this occurs because of considerations of capacity and economic (business) perspectives, rather than on the basis of the principle of network geometry. Consequently, there is a direct interest for large-scale central networks to have subsystems as a decentralized cluster included into the complex network.

Because of the principle of self-organization, it also offers the possibility and the guarantees for being able to make local decisions with respect to, for example, further-reaching sustainability without abandoning the principle of scale size ("economies of scale"). Procedurally, it implies that authorities and (public) grid managers may abandon policy aiming for a fixed ultimate goal.

Systems within decentralized planning concepts may lead to networks, complex or not, with a more strongly decentralized network structure with part of the networks performing relatively autonomously. These may support flexible planning concepts in town and country planning. Moreover, the issue of a more precise attribution of (network) costs to specific customers or transactions (which become more and more important as complexity decreases with ongoing liberalization) may be solved or may easier be solved. Concepts as such which support increasing flexibility can anticipate changing market developments. Moreover, it causes smaller investments with fewer risks in liberalized markets.

The effect of scale size by various technological developments has decreased the last 50 years, because of the low energy density and little purification efficiency per $\mathrm{m}^{2}$. This is also because improvements of energy transformation techniques and waste (water) transformation and purification have had relatively more influence on small-scale systems. The main technical advantage of (incorporating) decentralized systems is that, because of the scale size, the flows transported, processed or generated can be separated more easily into various qualities at source. In addition, the transport, the treatment, the use and/or the 
processing per subflow can take place more efficiently according to exergetic principles, such as cascading, where further optimization against user specifications becomes possible. A disadvantage is the difficult organization, maintenance, exploitation and inspection. The development of (legal) conditions with respect to responsibilities and periodic inspections is crucial for (decentralized) systems and determines a successful penetration of this technology.

Decentralized and local (sub)systems as parts of complex networks will possibly play an important role. It is important that each of the regional systems is connected "aristocratically" within higher scale levels, according to the principle of overlap as described in Christopher Alexander's 'semi-grid axiom'.

Network relationships support a process of so-called mutual innovation and, consequently, reduce the distances between central and decentralized solutions. In addition to this, there is the advantage of vicinity facilitates the "face-to-face" interaction and horizontal communication.

In order to accomplish the desired shift to a larger interest in future value and power of correction, small, gradually changing innovations should alternate (incrementally) with top-down innovation.

It is a problem that particularly the innovation related aspects come off worst because of a lack of control and grip, together with the aspects of maintenance and sustainability, in the current or intended liberalized situation of different essential subflows. The alternation of incremental and structural innovation appears to be the key to work out conflicting interests coherently for the short and the long term, without this leading to concession-driven solutions which are now quite common, and which lead to sustainability disappearing more and more or becoming only a fake type.

\section{Conclusion}

Redesigning large parts of the primary process in a top-down manner is necessary for the implementation of the substantial structural and sustainable improvements. Within the alternating process of incremental and structural innovation that was suggested above, the incremental innovation should shift more to solutions which follow the principle of subsidiarity of the European Community (Subsidiarity is the idea that matters should be handled by the lowest competent authority). It will lead to the desired larger differentiation in quality when there is less involvement, and it will support the main starting points of the European Community, and also (literally) the starting points of the wish for liberalizing the various utilities within the European market. Establishing the incremental innovation from the lowest scale levels may be a method for solving another topical problem on a European level, viz. the creation of equality for all member states, or, in other words, the accomplishment of a "Level Playing Field". In the set of demands of optimum flexibility, a smaller scale can guarantee better flexibility and units that are exchangeable to a larger degree.

As a conclusion, it can be stated that differentiation and flexibility in the area of town and country planning are preconditions for being able to anticipate 
uncertainties in the long term. Additionally, it is easier to anticipate dynamic developments that are characteristic of today's society. The process of urbanization and the infrastructural transport and distribution systems of the essential flows should be prevented from developing in separate ways. A sound, integral (Eu)regional planning based (first) on a combination of conventional (central) systems and additional decentralized systems (or, actually, the other way round), can prevent the risk of a possible "deadlock" of the current central systems, with all the accompanying health risks. The philosopher of culture Tom Lemaire claims "what matters is finding the right ratio between the global and the local. Local aspects should not be wiped out by globalization, but should get a new place". The strategic or random integration of decentralized clusters into the growing central networks, that approach autonomy as much as possible, will contribute to the increase of the robustness of these central (complex) networks, provided that the other conditions of scale invariance are met. Thus, it seems that the developing directions of "economy of scales" and "scale economy" need each other according to the principles of mutual interdependence.

\section{References}

[1] Alexander, Ch. The City is Not a Tree. Architectural Forum. nr.04 \& 05 / Design. nr.02. 1965.

[2] Frey, H.W. The search for a sustainable city. An account of current debate and research, PLEA 2004, The $21^{\text {st }}$ Conference on Passive and Low Energy Architecture. Eindhoven University of Technology. The Netherlands. 2004.

[3] Moet, D. Autarkie. Zelfvoorzienende woonwerklandschappen. Park. Haarlem. The Netherlands. 2004.

[4] McDonach, K. \& Yaneske, P.P. Environmental management systems and sustainable development, The Environmentalist, vol.22, pp217-226, Academic Publishers, Kluwer. 2002.

[5] Timmeren, A. van. The scale of Autarky; self sufficiency through integrated design of decentralised natural technologies in city districts and building clusters. Proceedings Sustainable Building 2002. Oslo. Norway. 2002.

[6] Timmeren, A. van, Kristinsson, J., Röling, L.C. Existing infrastructures: $a$ restriction for real sustainable development, in: 'The Sustainable City III; Urban Regeneration and Sustainability. Wessex Institute of Technology. WIT Press. Southampton. United Kingdom. 2004.

[7] Timmeren, A. van, Kristinsson, J., Röling, L.C. The interrelationship of sustainability and resilience- \& vulnerability of networks, related to the critical flows in society; a future deadlock?. Proceedings International Conference Sustainable Building (SB05). Tokyo. Japan. 2005.

[8] Watts, D.J., Strogatz, S.H., Collective dynamics of small world networks, Nature nr.393. p.440-442. 1998. 